\title{
Meilleures approximations diophantiennes d'un élément du tore $\mathbb{T}^{d}$
}

\author{
par \\ Nicolas Chevallier (Mulhouse)
}

1. Introduction. Le développement en fraction continue d'un réel $\theta=$ $\left[0, a_{1}, \ldots, a_{n}, \ldots\right]$ appartenant à l'intervalle $[0,1]$ vérifie trois propriétés fondamentales.

(1) Soit $T$ l'application $x \in] 0,1] \mapsto\{1 / x\} \quad(\{1 / x\}$ désigne la partie fractionnaire de $1 / x)$. Pour tout entier $n \geq 1$ on a

$$
a_{n}=\left[T^{n}(x)\right]
$$

( $\left[T^{n}(x)\right]$ désigne la partie entière de $\left.T^{n}(x)\right)$.

(2) (L'unimodularité) Deux réduites consécutives $p_{n} / q_{n}$ et $p_{n+1} / q_{n+1}$ satisfont

$$
\operatorname{det}\left(\begin{array}{cc}
p_{n} & p_{n+1} \\
q_{n} & q_{n+1}
\end{array}\right)= \pm 1
$$

(3) (La propriété de meilleure approximation) Une fraction $a / b$ est une réduite de $x$ si et seulement si

$$
\forall p \in \mathbb{Z}, \forall q \in\{1, \ldots, b-1\}, \quad|b x-a|<|q x-p| .
$$

La définition d'un développement en fractions continues multidimensionnel privilégie certaines de ces propriétés. L'algorithme de Jacobi-Perron est le premier exemple de développement en fractions continues multidimensionnel, son point de départ est l'application $(x, y) \in[0,1] \times] 0,1] \mapsto$ $(\{1 / y\},\{x / y\})$, il vérifie la propriété d'unimodularité mais il ne possède pas la propriété de meilleure approximation. De nombreux autres algorithmes ont été proposés, en général ils sont unimodulaires mais ne vérifient pas la propriété de meilleure approximation. Lagarias a montré que ces deux propriétés sont incompatibles en dimension $2([4])$. Il est le premier à avoir étudié de manière systématique la suite des meilleures approximations d'un

2000 Mathematics Subject Classification: 11J13, 11J70, 11J83. 
élément du tore $\mathbb{T}^{d}=(\mathbb{R} / \mathbb{Z})^{d}([4]-[7])$. Ce travail se situe dans la même perspective et a pour objectif :

1) étendre les théorèmes métriques de Khinchin et Borel-Bernstein sur la croissance des dénominateurs des réduites et des coefficients du développement en fraction continue d'un réel,

2) étudier les extensions de la minoration $q_{n+1}\left|q_{n} \theta-p_{n}\right| \geq 1 / 2$ en dimension $d \geq 2$.

1.1. Notations. Soit $d$ un entier; munissons $\mathbb{R}^{d}$ d'une norme $\|\cdot\|$ et $\mathbb{T}^{d}$ de la distance quotient. Nous utiliserons la notation standard $\|\theta\|$ pour désigner la distance dans $\mathbb{T}^{d}$, de 0 à $\theta .|A|$ désigne la mesure de Lebesgue d'une partie $A$ de $\mathbb{R}^{d}$ où $\mathbb{T}^{d}$.

Si $A$ est une partie d'un espace métrique $(X, d)$, appelons

$$
r(A)=\inf \{d(x, y): x \neq y, x, y \in A\}, \quad e(A)=\sup \{d(x, A): x \in X\} .
$$

Lorsque $\theta \in \mathbb{T}^{d}$ notons

$$
r(n, \theta)=r(\{0, \theta, \ldots, n \theta\}), \quad e(n, \theta)=e(\{0, \theta, \ldots, n \theta\}) .
$$

Soient $G$ un groupe et $A$ une partie ou un élément de $G ;\langle A\rangle$ désigne le sous-groupe engendré par $A$.

Soient $m$ et $n$ deux entiers ; $m \wedge n$ désigne le plus grand diviseur commun de $m$ et $n$.

Soient $n$ et $k$ deux entiers; $d(n), \sigma_{k}(n)$, et $\phi(n)$ désignent les fonctions arithmétiques usuelles, c'est-à-dire

$$
d(n)=\sum_{q \mid n} 1, \quad \sigma_{k}(n)=\sum_{q \mid n} q^{k}, \quad \phi(n)=\sum_{q \wedge n=1, q \leq n} 1 .
$$

1.2. Définition des meilleures approximations et des coefficients

DÉfinition 1.1. Soit $\theta$ un élément de $\mathbb{T}^{d}$. Une meilleure approximation de $\theta$ est un entier $q$ supérieur ou égal à 1 tel que $\|q \theta\|<\|k \theta\|$ pour tous les entiers $k$ compris entre 1 et $q-1$.

Soit $\theta$ un élément de $\mathbb{R}^{d}$. Un entier $q$ est une meilleure approximation de $\theta$ si $q$ est une meilleure approximation de la projection de $\theta$ dans $\mathbb{T}^{d}$.

Ordonnons par ordre croissant la suite des meilleures approximations. Posons $q_{0}(\theta)=1$ et désignons par $q_{n}(\theta)$ la $n+1$-ième meilleure approximation de $\theta$ si elle existe. Dans la suite, l'écriture $q_{n}(\theta)$ suppose implicitement que $q_{n}(\theta)$ existe. La notation

$$
r_{n}(\theta)=\left\|q_{n}(\theta) \theta\right\|
$$

sera utilisée dans tout le texte et lorsqu'il n'y pas d'ambiguïté nous écrirons simplement $q_{n}$ et $r_{n}$. 
Par définition des meilleures approximations les boules ouvertes du tore $B\left(k \theta, r_{n}(\theta) / 2\right), k=0, \ldots, q_{n+1}(\theta)-1$, sont disjointes. Lorsque $\mathbb{R}^{d}$ est muni de la norme sup, l'inégalité fondamentale

$$
q_{n+1}(\theta) r_{n}^{d}(\theta) \leq 1
$$

(cf. [1]) se déduit facilement de cette observation.

Remarque 1 . Soit $\theta$ un élément de $\mathbb{R}^{d}$. La suite des meilleures approximations de $\theta$ est infinie si et seulement si $\theta \notin \mathbb{Q}^{d}$. D'autre part, des exemples simples montrent que la suite des meilleures approximations dépend de la norme choisie.

Soit $x=\left[a_{0}, \ldots, a_{n}, \ldots\right]$ un réel. Les relations

$$
a_{n}=\left[\frac{q_{n}(x)}{q_{n-1}(x)}\right]=\left[\frac{r_{n-2}(x)}{r_{n-1}(x)}\right]
$$

suggèrent deux manières de définir les coefficients d'un élément $\theta$ de $\mathbb{T}^{d}$,

$$
a_{n}(\theta)=\frac{q_{n}(\theta)}{q_{n-1}(\theta)} \quad \text { et } \quad b_{n}(\theta)=\left(\frac{r_{n-2}(\theta)}{r_{n-1}(\theta)}\right)^{d} .
$$

L'exposant $d$ est naturel car $q_{n}(\theta) r_{n-1}^{d}(\theta)$ est proportionnel à la mesure de la réunion des boules $B\left(k \theta, r_{n-1}(\theta) / 2\right), k=0, \ldots, q_{n}(\theta)-1$. Le quotient $a_{n} / b_{n}$ représente donc le rapport des mesures de deux régions du tore. Lorsque ces régions ont une mesure de l'ordre de 1 , c'est-à-dire lorsque $q_{n}(\theta) r_{n-1}^{d}(\theta)$ et $q_{n-1}(\theta) r_{n-2}^{d}(\theta)$ ne sont pas trop petits, les coefficients $a_{n}$ et $b_{n}$ sont du même ordre de grandeur.

1.3. Croissance de meilleures approximations et des coefficients. Supposons $d \geq 2$ et $\mathbb{R}^{d}$ muni de la norme sup.

Lagarias a montré que la croissance de la suite $\left(q_{n}(\theta)\right)_{n \in \mathbb{N}}$ est au moins exponentielle pour les éléments $\theta$ du tore $\mathbb{T}^{d}$ dont les coordonnées ne sont pas toutes rationnelles ([5]). En dimension 1, Khinchin a montré que la croissance de la suite $\left(q_{n}(x)\right)_{n \in \mathbb{N}}$ est au plus exponentielle pour presque tous les $x$ de $\mathbb{T}^{1}$. Cette majoration s'étend aux dimensions supérieures :

ThÉORÈme 1. Il existe une constante $C$ ne dépendant que de la dimension, telle que pour presque tout $\theta \in \mathbb{T}^{d}$, et pour $n$ assez grand, $q_{n}(\theta) \leq C^{n}$.

Ce théorème sera utile pour notre objectif principal qui est d'étendre le résultat de Borel-Bernstein sur la croissance des coefficients du développement en fraction continue monodimensionnel :

Désignons par $\left(a_{n}\right)_{n \geq 0}$ la suite des coefficients du développement en fraction continue du réel $x, x=\left[a_{0}, a_{1}, \ldots\right]$. Soient $g$ et $h$ deux applications de $\mathbb{N}$ dans $\mathbb{R}^{+}$telles que $\sum_{n \geq 0} g(n)<\infty$ et $\sum_{n \geq 0} h(n)=\infty$. 
1) Pour presque tout réel $x$ il existe au plus un nombre fini de $n$ tels que

$$
a_{n} \geq 1 / g(n) .
$$

2) Pour presque tout réel $x$ il existe une infinité de $n$ tels que

$$
a_{n} \geq 1 / h(n) .
$$

ThÉORÈme 2 . Soit $g$ et $h$ deux applications décroissantes de $\mathbb{N}$ dans $\mathbb{R}^{+}$ telles que $\sum_{n \geq 0} g(n)<\infty$ et $\sum_{n \geq 0} h(n)=\infty$.

1) Pour presque tout $\theta \in \mathbb{T}^{d}$ il existe au plus un nombre fini de $n$ tels que

$$
b_{n+1}(\theta)=\left(\frac{r_{n-1}(\theta)}{r_{n}(\theta)}\right)^{d} \geq \frac{1}{g(n)} .
$$

2) Pour presque tout $\theta \in \mathbb{T}^{d}$ il existe une infinité de $n$ tels que

$$
b_{n+1}(\theta)=\left(\frac{r_{n-1}(\theta)}{r_{n}(\theta)}\right)^{d} \geq \frac{1}{h(n)} .
$$

En dimension 1, l'application $T: x \in] 0,1] \mapsto\{1 / x\} \in[0,1]$ rend inutile l'hypothèse de monotonie sur $g$ et $h$. En dimension $d \geq 2$, un résultat intermédiaire remplace l'application $T$ :

ThÉORÈme 3 . Soit $g$ et $h$ deux applications décroissantes de $\mathbb{N}$ dans $\mathbb{R}^{+}$ telles que $\sum_{n \geq 0} g(n)<\infty$ et $\sum_{n \geq 0} h(n)=\infty$.

1) Pour presque tout $\theta \in \mathbb{T}^{d}$ il existe au plus un nombre fini d'entiers $n$ tels que

$$
\left(\frac{r_{n-1}(\theta)}{r_{n}(\theta)}\right)^{d} \geq \frac{1}{q_{n}(\theta) g\left(q_{n}(\theta)\right)} .
$$

2) Pour presque tout $\theta \in \mathbb{T}^{d}$ il existe une infinité d'entiers $n$ tels que

$$
\left(\frac{r_{n-1}(\theta)}{r_{n}(\theta)}\right)^{d} \geq \frac{1}{q_{n}(\theta) h\left(q_{n}(\theta)\right)} .
$$

Pour $d=1$, ce théorème se déduit facilement du théorème métrique de Khinchin sur les approximations diophantiennes et de l'inégalité $q_{n}\left|q_{n} x-p_{n}\right|$ $\geq 1 / 2$. Mais lorsque $d \geq 2$ les résultats de la sous-section 1.4 montrent que cette inégalité n'admet pas d'analogue satisfaisant. Il faut donc faire une démonstration directe du théorème précédent.

Les coefficients $\left(a_{n}\right)$ et $\left(b_{n}\right)$ sont liés par l'inégalité facile à prouver

$$
\frac{q_{n}(\theta)}{q_{n-1}(\theta)} \geq \frac{r_{n-2}(\theta)-r_{n}(\theta)}{r_{n-1}(\theta)}
$$

(cf. [1]), d'où le corollaire : 
Corollaire 1. Pour presque tout $\theta$ appartenant à $\mathbb{T}^{d}$ la suite $q_{n+1} / q_{n}$ n'est pas bornée.

1.4. Minoration de $q_{n}(\theta) r_{n-1}^{d}(\theta)$. Soit $x \in \mathbb{T}^{1}$. La relation $q_{n-1}(x) r_{n}(x)$ $+q_{n}(x) r_{n-1}(x)=1$ montre que

$$
q_{n}(x) r_{n-1}(x) \geq 1 / 2 .
$$

Cette inégalité a de nombreuses applications, en particulier elle fournit une démonstration simple en dimension 1 du théorème 3 . En dimension $d=2$ la situation est plus complexe (cf. [1] et [2]) car :

- Il existe $\theta \in \mathbb{T}^{2}$ tel que $\lim _{n \rightarrow \infty} q_{n}(\theta) r_{n-1}^{2}(\theta)=0$.

- Soit $\theta \in \mathbb{T}^{2}$. Si $\mathbb{Z} \theta$ est dense dans $\mathbb{T}^{2}$ alors il existe une infinité d'entiers $n$ tels que $q_{n}(\theta) r_{n-1}(\theta) r_{n-2}(\theta) \geq 1 / 100$.

- L'ensemble des $\theta$ appartenant à $\mathbb{T}^{2}$ tels que $\mathbb{Z} \theta$ soit dense dans $\mathbb{T}^{2}$ et

$$
\liminf _{n \rightarrow \infty} q_{n}(\theta) r_{n-1}(\theta) r_{n-2}(\theta)=0,
$$

contient un $G_{\delta}$ dense.

En dimension $d=3$ l'analogue de la deuxième affirmation est faux :

Proposition 1.1. Il existe $\theta \in \mathbb{T}^{3}$ tel que $\mathbb{Z} \theta$ soit dense dans $\mathbb{T}^{3}$ et

$$
\lim _{n \rightarrow \infty} q_{n}(\theta) r_{n-1}(\theta) r_{n-2}(\theta) r_{n-3}(\theta)=0 .
$$

Donnons maintenant des inégalités presque sûres.

THÉORÈme 4. Il existe une constante $c>0$ ne dépendant que de la dimension telle que pour presque tout $\theta$ de $\mathbb{T}^{d}$ on ait

$$
\limsup _{n \rightarrow \infty} q_{n}(\theta) r_{n-1}^{d}(\theta) \geq c .
$$

ThÉORÈme 5. Pour presque tout $\theta$ de $\mathbb{T}^{d}$ on a

$$
\liminf _{n \rightarrow \infty} q_{n}(\theta) r_{n-1}^{d}(\theta)=0 .
$$

Combiné avec les inégalités sur les minima successifs d'un réseau le théorème 4 a pour conséquence :

Corollaire 2. Il existe une constante $C$ ne dépendant que de la dimension telle que pour presque tout $\theta$ de $\mathbb{T}^{d}$ il existe une infinité de $n$ tels que $e(n, \theta) \leq C r(n, \theta)$.

Ce corollaire renforce un résultat de [1] dans lequel la constante $C$ dépend de $\theta$. Un passage au complémentaire et un principe de transfert entre approximations diophantiennes homogènes et non homogènes simplifient notablement la démonstration que nous avions donnée dans [1]. Mais une amélioration de la méthode de [1] conduit à une constante $C$ indépendante de $\theta$. 
2. Démonstration du théorème 1. Ce théorème repose sur une estimation remarquable de W. M. Schmidt ([8], p. 61) qui contient le théorème métrique de Khinchin sur les approximations diophantiennes simultanées :

Soit $\delta$ une application décroissante de $\mathbb{N}^{*}$ dans $\mathbb{R}^{+}$telle que $\sum_{n>0} \delta(n)$ $=\infty$. Posons

$$
\Delta(n)=\sum_{1 \leq k \leq n} \delta(k), \quad \Omega(n)=\sum_{1 \leq k \leq n} \delta(k) / k
$$

et, pour $\theta \in \mathbb{T}^{d}$,

$$
N(\theta, n)=\operatorname{card}\left(\left\{1 \leq k \leq n:\|k \theta\|^{d} \leq \delta(k)\right\}\right) .
$$

Pour $\varepsilon>0$,

$$
N(\theta, n)=2^{d} \Delta(n)+O\left(\Delta(n)^{1 / 2} \Omega(n)^{1 / 2}(\ln \Delta(n))^{2+\varepsilon}\right)
$$

pour presque tout $\theta$ de $\mathbb{T}^{d}$.

Utilisons l'estimation précédente avec $\delta(n)=1 / n$. Pour presque tout $\theta$ il existe un entier $n_{0}(\theta)$ tel que

$$
\forall n \geq n_{0}, \quad N(\theta, n) \leq 2^{d+1} \ln n .
$$

Minorons $N\left(\theta, q_{n}(\theta)\right)$ pour $\theta \in \mathbb{T}^{d}$.

Soit $p$ est un entier compris entre 1 et $\left(q_{k+1}(\theta) / q_{k}(\theta)\right)^{1 /(d+1)}$ et $q=$ $p q_{k}(\theta)$. Comme $q_{k+1}(\theta) r_{k}(\theta)^{d} \leq 1$ nous avons

$$
q\|q \theta\|^{d} \leq q_{k}(\theta) p^{d+1}\left\|q_{k}(\theta) \theta\right\|^{d} \leq q_{k+1}(\theta) r_{k}(\theta)^{d} \leq 1,
$$

et donc

$$
\begin{aligned}
N\left(\theta, q_{n}(\theta)\right) & \geq \sum_{0 \leq k \leq n-1}\left[\left(\frac{q_{k+1}(\theta)}{q_{k}(\theta)}\right)^{1 /(d+1)}\right] \\
& \geq \sum_{0 \leq k \leq n-1}\left(\frac{q_{k+1}(\theta)}{q_{k}(\theta)}\right)^{1 /(d+1)}-n .
\end{aligned}
$$

La moyenne arithmétique est supérieure à la moyenne géométrique, donc

$$
N\left(\theta, q_{n}(\theta)\right) \geq n\left(q_{n}^{1 /(n(d+1))}(\theta)-1\right) .
$$

D'où, pour presque tout $\theta$,

$$
q_{n}(\theta) \geq n_{0}(\theta) \Rightarrow 2^{d+1} \ln q_{n}(\theta) \geq n\left(q_{n}^{1 /(n(d+1))}(\theta)-1\right) .
$$

En posant $q_{n}(\theta)=\exp \left(n A_{n}\right)$, nous obtenons

$$
2^{d+1} A_{n} \geq \exp \left(A_{n} /(d+1)\right)-1
$$

et donc $A_{n} \leq C(d)$ où $C(d)$ ne dépend que de $d$. 
3. Un lemme sur la répartition des sous-groupes de $\mathbb{T}^{d}$. Munissons $\mathbb{Z} / n \mathbb{Z}$ de la distance quotient, i.e.

$$
d(a, b)=\inf \{|x-y|: x \in a \text { et } y \in b\} .
$$

Soit $d$ un entier supérieur ou égal à 2 . Munissons $(\mathbb{Z} / n \mathbb{Z})^{d}$ de la distance

$$
d\left(\left(a_{1}, \ldots, a_{d}\right),\left(b_{1}, \ldots, b_{d}\right)\right)=\sup \left\{d\left(a_{i}, b_{i}\right): i \in\{1, \ldots, d\}\right\} .
$$

Lemme 1. Soient $n$ un entier, $d$ un entier supérieur ou égal à 2 et $\alpha$ un réel appartenant à l'intervalle ]0,1]. Posons

$$
B(n)=\left\{a=\left(a_{1}, \ldots, a_{d}\right) \in(\mathbb{Z} / n \mathbb{Z})^{d}: \operatorname{ordre}\left(a_{1}\right)=n, r(\langle a\rangle) \geq \alpha n^{1-1 / d}\right\} .
$$

On $a$

$$
\operatorname{card}(B(n)) \geq \phi(n)\left\{n^{d-1}-2^{2 d-1} \alpha^{d} n^{d-2} \sigma_{1}(n)\right\} .
$$

Démonstration. La démonstration de ce lemme est une simple adaptation du cas $d=2$ qui a été prouvé dans [1]. Identifions $(\mathbb{Z} / n \mathbb{Z})^{d}$ et $\mathbb{Z} / n \mathbb{Z} \times(\mathbb{Z} / n \mathbb{Z})^{d-1}$. Notons

$$
\begin{aligned}
\bar{B}(n)=\{z=(x, y) \in & (\mathbb{Z} / n \mathbb{Z})^{d}: \\
& \left.\operatorname{ordre}(x)=n,\langle(x, y)\rangle \cap B\left(0, \alpha n^{1-1 / d}\right) \neq\{0\}\right\} .
\end{aligned}
$$

Nous avons

$$
\operatorname{card}(B(n))=n^{d-1} \phi(n)-\operatorname{card}(\bar{B}(n)) .
$$

Majorons le cardinal de $\bar{B}(n)$. Soit $x$ un élément de $\mathbb{Z} / n \mathbb{Z}$ d'ordre $n$. Posons

$$
m(x)=\left\{y \in(\mathbb{Z} / n \mathbb{Z})^{d-1}:\langle(x, y)\rangle \cap B\left(0, \alpha n^{1-1 / d}\right) \neq\{0\}\right\} .
$$

Nous avons

$$
\begin{aligned}
y \in m(x) & \Leftrightarrow \exists(a, b) \in B\left(0, \alpha n^{1-1 / d}\right) \backslash\{0\}, \exists k \in \mathbb{Z}, a=k x \text { et } b=k y \\
& \Leftrightarrow \exists(a, b) \in B\left(0, \alpha n^{1-1 / d}\right) \backslash\{0\}, b=\left(x^{-1} a\right) y=a\left(x^{-1} y\right),
\end{aligned}
$$

donc $m(x)$ est en bijection avec

$$
M=\left\{z \in(\mathbb{Z} / n \mathbb{Z})^{d-1}: \exists(a, b) \in B\left(0, \alpha n^{1-1 / d}\right) \backslash\{0\}, b=a z\right\} .
$$

Par conséquent $\operatorname{card}(\bar{B}(n))=\phi(n) \operatorname{card}(M)$.

Il nous faut majorer le cardinal de $M$. Associons à chaque $(a, b) \in$ $B\left(0, \alpha n^{1-1 / d}\right) \backslash\{0\}$ l'ensemble $E(a, b)=\left\{z \in(\mathbb{Z} / n \mathbb{Z})^{d-1}: b=a z\right\}$. Nous avons

$$
\operatorname{card}(M) \leq \sum_{(a, b) \in B\left(0, \alpha n^{1-1 / d}\right) \backslash\{0\}} \operatorname{card}(E(a, b)) .
$$

Soit $(a, b) \in \mathbb{Z} / n \mathbb{Z} \times(\mathbb{Z} / n \mathbb{Z})^{d-1}$ tel que $a$ soit d'ordre $p$. Le nombre de solutions de l'équation $b=a z$ est $(n / p)^{d-1}$ si $b \in(\langle a\rangle)^{d-1}$ et 0 si $b \notin$ $(\langle a\rangle)^{d-1}$. Comme $\langle a\rangle=\langle n / p\rangle$, le nombre de $b \in(\langle a\rangle)^{d-1}$ dont la distance à 0 
est inférieure à $\alpha n^{1-1 / d}$ est $\left(2\left[\alpha n^{1-1 / d} /(n / p)\right]+1\right)^{d-1}$. Ainsi le nombre de couples $(a, b) \in B\left(0, \alpha n^{1-1 / d}\right) \backslash\{0\}$ tels que $a$ soit d'ordre $d$ et $b \in(\langle a\rangle)^{d-1}$, est

$$
2\left[\alpha n^{1-1 / d} /(n / p)\right]\left(2\left[\alpha n^{1-1 / d} /(n / p)\right]+1\right)^{d-1} .
$$

Comme $\left[\alpha n^{1-1 / d} /(n / p)\right]$ est un nombre entier nous avons

$$
\begin{aligned}
2\left[\alpha n^{1-1 / d} /(n / p)\right]\left(2\left[\alpha n^{1-1 / d} /(n / p)\right]+1\right)^{d-1} & \\
\leq & 2\left[\alpha n^{1-1 / d} /(n / p)\right]\left(4\left[\alpha n^{1-1 / d} /(n / p)\right]\right)^{d-1} .
\end{aligned}
$$

Finalement, nous obtenons

$$
\begin{aligned}
\operatorname{card}(M) & \leq \sum_{p \mid n}(n / p)^{d-1} 2^{2 d-1} \alpha^{d}\left(n^{-1 / d} p\right)^{d} \\
& \leq 2^{2 d-1} \alpha^{d} n^{d-2} \sum_{p \mid n} p=2^{2 d-1} \alpha^{d} n^{d-2} \sigma_{1}(n)
\end{aligned}
$$

4. Démonstration du théorème 3. La démonstration de ce théorème est une adaptation d'une méthode classique. Le principe de base est exposé dans le livre de Sprindžuk ([9], pages 18 à 28).

1) Si $r \in[0,1 / 2]$ la mesure de l'ensemble des $\theta \in \mathbb{T}^{d}$ tels que $\|q \theta\| \leq r$ est $(2 r)^{d}$. Par conséquent, d'après le lemme de Borel-Cantelli pour presque tout $\theta \in \mathbb{T}^{d}$ il existe un nombre fini d'entiers $q$ tels que $\|q \theta\| \leq(g(q))^{1 / d}$. Ainsi $r_{n}^{d}(\theta)>g\left(q_{n}(\theta)\right)$ pour $n$ assez grand. Or $q_{n}(\theta) r_{n-1}^{d}(\theta) \leq 1$, donc

$$
\left(\frac{r_{n-1}(\theta)}{r_{n}(\theta)}\right)^{d} \leq \frac{1}{q_{n}(\theta) g\left(q_{n}(\theta)\right)}
$$

2) Préliminaire sur les séries. Si $h$ ne vérifie pas $\lim _{n \rightarrow \infty} n h(n)=0$, posons $\widetilde{h}(n)=\min (h(n), 1 / n)$. La fonction $\widetilde{h}$ est décroissante et $\sum_{n \geq 0} \widetilde{h}(n)$ $=\infty$ car

$$
\begin{aligned}
\left(\widetilde{h} \text { décroissante et } \sum_{n \geq 0} \widetilde{h}(n)<\infty\right) & \Rightarrow \lim _{n \rightarrow \infty} n \widetilde{h}(n)=0 \\
& \Rightarrow \widetilde{h}(n)=h(n) \text { pour } n \text { assez grand } \\
& \Rightarrow \sum_{n \geq 0} h(n)<\infty .
\end{aligned}
$$

La suite $S_{n}=\sum_{p=0}^{n} \widetilde{h}(p)$ tend vers l'infini quand $n \rightarrow \infty$, donc $S_{n}^{-1 / 2} n \widetilde{h}(n)$ tend vers 0 . De plus $S_{n}^{-1 / 2} \widetilde{h}(n)$ décroît avec $n$ et $\sum_{n \geq 0} S_{n}^{-1 / 2} \widetilde{h}(n)=\infty$ car

$$
\sum_{n=1}^{N} S_{n}^{-1 / 2} \widetilde{h}(n) \geq \sum_{n=1}^{N} \frac{S_{n}-S_{n-1}}{S_{n}^{1 / 2}+S_{n-1}^{1 / 2}}=\sum_{n=1}^{N}\left(S_{n}^{1 / 2}-S_{n-1}^{1 / 2}\right)=S_{N}^{1 / 2}-S_{0}^{1 / 2} .
$$


La fonction $H(n)=S_{n}^{-1 / 2} \widetilde{h}(n)$ vérifie les conditions :

(a) $H$ est décroissante et $H(n) \leq h(n)$ pour $n$ assez grand,

(b) $\sum_{n \geq 0} H(n)=\infty$,

(c) $\lim _{n \rightarrow \infty} n H(n)=0$.

Il suffit de prouver le théorème avec la fonction $H$ à la place de $h$. Posons $f(n)=(n H(n))^{1 / d}$. Alors $f(n)$ tend vers 0 quand $n$ tend vers l'infini et $\sum_{n>0} f(n)^{d} / n=\infty$. Enfin, quitte à multiplier $H$ par un réel suffisamment petit, on peut supposer que $f(n) \leq 1 / 2$ pour tout $n \in \mathbb{N}$.

Notons $T$ l'endomorphisme défini par $T: x \in \mathbb{T}^{d} \mapsto 2 x \in \mathbb{T}^{d}$. Soit $\alpha$ un réel strictement positif qui sera choisi plus loin. Pour $n \in \mathbb{N}^{*}$, soient

$$
\begin{aligned}
G(n) & =\left\{x \in \mathbb{T}^{d}: n x=0\right\}, \\
B(n) & =\left\{a \in G(n): \operatorname{ordre}(a)=n, r(\langle a\rangle) \geq \alpha n^{-1 / d}\right\}, \\
E(n) & =\left\{x \in \mathbb{T}^{d}: d(x, B(n)) \leq f(n) n^{-1-1 / d}\right\}, \\
E^{\prime}(n) & =\left\{x \in \mathbb{T}^{d}: d(x, B(n)) \leq 2 f(n) n^{-1-1 / d}\right\} .
\end{aligned}
$$

Appelons $A$ l'ensemble des $\theta$ de $\mathbb{T}^{d}$ appartenant à une infinité de $E(n)$ avec $n$ impair, $A^{\prime}$ l'ensemble des $\theta$ de $\mathbb{T}^{d}$ appartenant à une infinité de $E^{\prime}(n)$ avec $n$ impair et $\mathcal{A}$ l'ensemble des $\theta \in \mathbb{T}^{d}$ pour lesquels il existe une infinité de meilleures approximations consécutives $q_{n-1}<q_{n}$ telles que $r\left(q_{n}, \theta\right) \leq(2 / \alpha) f\left(q_{n}\right) r\left(q_{n-1}, \theta\right)$. Nous devons prouver que $\mathcal{A}$ est de mesure 1 ; pour cela nous montrons successivement que :

(1) $A \subset \mathcal{A}$,

(2) $A$ est de mesure 0 ou 1 ,

(3) $A$ est de mesure strictement positive.

Notons que la décroissance de $H$ est utile pour effectuer des transformations d'Abel dans l'étape (3).

Preuve de (1). Soit $x \in E(n)$. Si $y \in B(n)$ est tel que l'on a $d(x, y) \leq$ $f(n) n^{-1-1 / d}$, alors $\|n x\| \leq f(n) n^{-1 / d}$ car $n y=0$. Pour tout $m<n$ nous avons

$$
\begin{aligned}
\|m x\| & =\|m(y+x-y)\| \geq\|m y\|-\|m(x-y)\| \geq \alpha n^{-1 / d}-m\|x-y\| \\
& \geq \alpha n^{-1 / d}-f(n) n^{-1 / d} \geq(\alpha-f(n))\{\|n x\| / f(n)\} .
\end{aligned}
$$

Comme $f(n) \rightarrow 0$ quand $n \rightarrow \infty$, pour $n$ assez grand nous avons

$$
\|m x\| \geq\left(\frac{\alpha}{2}\right) \frac{\|n x\|}{f(n)} .
$$

Ainsi $n$ est une meilleure approximation de $x$. Appelons $m$ la meilleure approximation qui précède $n$. Nous avons

$$
\frac{r(m, x)}{r(n, x)} \geq \frac{\alpha}{2 f(n)}
$$


Preuve de (2). Le lemme suivant se trouve dans [9], page 21; il repose essentiellement sur le théorème de densité de Lebesgue.

Lemme 2. Soient $\left(B_{k}\right)$ une suite de boules de $\mathbb{R}^{d}$ muni de la norme sup et $\delta$ un nombre strictement positif. Supposons que $\operatorname{diam}\left(B_{k}\right)$ tende vers 0 quand $k$ tend vers l'infini. Si $\left(C_{k}\right)$ est une suite de parties de $\mathbb{R}^{d}$ telles que

$$
C_{k} \subset B_{k} \quad \text { et } \quad\left|C_{k}\right| \geq \delta\left|B_{k}\right|
$$

alors limsup $\sup _{k \rightarrow \infty} B_{k}=\limsup _{k \rightarrow \infty} C_{k}$ à un ensemble de mesure nulle près.

Utilisons le lemme pour montrer que $A$ est de mesure 0 ou 1.

Lorsque $n$ est impair l'application $T$ envoie $B(n)$ dans lui-même et $E(n)$ dans $E^{\prime}(n)$, donc $T(A) \subset A^{\prime}$. L'ensemble $B(n)$ est fini donc $E(n)$ est la réunion finie des boules $B\left(y, f(n) n^{-1-1 / d}\right), y \in B(n)$, la limite supérieure des $E(n)$ est donc la même que celle des boules qui composent $E(n)$. Le même résultat est valable pour $E^{\prime}(n)$. Par conséquent, d'après le lemme précédent, $A$ et $A^{\prime}$ diffèrent d'un ensemble de mesure nulle et comme $T$ est ergodique et conserve la mesure, $A$ est de mesure 0 ou 1.

Preuve de (3). Quelques lemmes sont nécessaires.

Lemme 3 ([9], p. 17). Soit $(\Omega, \mathcal{T}, \mu)$ un espace mesuré de mesure totale finie. Si $\left(A_{p}\right)$ est une suite de parties mesurables telles que

$$
\sum_{q=1}^{\infty} \mu\left(A_{q}\right)=\infty
$$

alors

$$
\mu\left(\limsup _{n \rightarrow \infty} A_{n}\right) \geq \limsup _{m \rightarrow \infty} \frac{\left(\sum_{1 \leq q \leq m} \mu\left(A_{q}\right)\right)^{2}}{\sum_{1 \leq p, q \leq m} \mu\left(A_{p} \cap A_{q}\right)} .
$$

Lemme 4 ([9], p. 52). Pour $r$ entier notons $T_{r}$ l'application $x \mapsto r x$ de $\mathbb{T}^{1}$ dans lui même. Soient $A$ et $B$ deux intervalles de $\mathbb{T}^{1}$, et $p$ et $q$ deux entiers. On a

$$
\left|T_{p}^{-1} A \cap T_{q}^{-1} B\right|=|A| \cdot|B|+O\left(|A| \frac{p \wedge q}{p}\right)
$$

où la constante implicite $d u O(.$.$) ne dépend ni des intervalles A$ et $B, n i$ des entiers $p$ et $q$.

Lemme 5. Soit $k$ un entier supérieur ou égal à 1 . On a

$$
\begin{gathered}
\sum_{1 \leq n \leq N} \sigma_{k}(n)=O\left(N^{k+1}\right), \sum_{\substack{1 \leq n \text { impair } \leq N \\
1 \leq n \text { impair } \leq N}} \phi(n) \sim c N^{2}, \\
\sum^{k} \phi(n) \geq A N^{k+2}
\end{gathered}
$$

où $A$ et $c$ sont des constantes strictement positives ne dépendant pas de $N$. 
La première estimation est standard (cf. [3]) et la troisième se déduit facilement de la seconde. La seconde inégalité se montre en adaptant la démonstration de l'estimation classique $\sum_{1 \leq n \leq N} \phi(n) \sim\left(3 / \pi^{2}\right) N^{2}$.

Convention. Dans toute la suite du paragraphe 4 les indices de sommation sont supposés ne prendre que des valeurs impaires.

Appliquons le lemme 3 à la suite $\left(A_{n}=E(n)\right)$. Il suffit de prouver

$$
\sum_{p \geq 1}|E(p)|=\infty \text { et } \sum_{1 \leq p, q \leq N}|E(p) \cap E(q)| \leq C\left\{\sum_{1 \leq p \leq N}|E(p)|\right\}^{2}
$$

où $C$ ne dépend pas de $N$.

1) D'après le lemme 1 , nous avons

$$
\operatorname{card}(B(p)) \geq \phi(p)\left(p^{d-1}-2^{2 d-1} \alpha^{d} p^{d-2} \sigma_{1}(p)\right),
$$

d'où

$$
\begin{aligned}
\sum_{1 \leq p \leq N}|E(p)| & \geq \sum_{1 \leq p \leq N} \operatorname{card}(B(p))\left(2 f(p) p^{-1-1 / d}\right)^{d} \\
& \geq \sum_{1 \leq p \leq N} f(p)^{d} p^{-1-d}\left(p^{d-1} \phi(p)-2^{2 d-1} \alpha^{d} p^{d-2} \sigma_{1}(p) \phi(p)\right)
\end{aligned}
$$

et comme $\phi(p) \leq p$, nous obtenons

$$
\begin{aligned}
\sum_{1 \leq p \leq N}|E(p)| & \geq \sum_{1 \leq p \leq N} f(p)^{d} p^{-1-d}\left(\phi(p) p^{d-1}-2^{2 d-1} \alpha^{d} p^{d-1} \sigma_{1}(p)\right) \\
& \geq \sum_{1 \leq p \leq N} f(p)^{d} p^{-d}\left(p^{d-2} \phi(p)-2^{2 d-1} \alpha^{d} p^{d-2} \sigma_{1}(p)\right) .
\end{aligned}
$$

Posons

$$
D(q)=\left(\frac{f(q)}{q}\right)^{d}-\left(\frac{f(q+2)}{q+2}\right)^{d}=\frac{H(q)}{q^{d-1}}-\frac{H(q+2)}{(q+2)^{d-1}}
$$

(nous ne considèrons que les $q$ impairs),

$$
G(q)=q^{d-2} \phi(q)-2^{2 d-1} \alpha^{d} q^{d-2} \sigma_{1}(q), \quad S(p)=\sum_{1 \leq q \leq p} G(q), \quad S(-1)=0 .
$$

Une transformation d'Abel montre que

$$
\sum_{1 \leq p \leq N} f(p)^{d} p^{-d} G(p)=\sum_{1 \leq p \leq N-2} S(p) D(p)+f\left(N^{\prime}\right) N^{\prime-d} S\left(N^{\prime}\right)
$$

où $N^{\prime}=N-1$ ou $N$. D'après le lemme 5 , nous avons $S(p) \geq A p^{d}-\alpha^{d} O\left(p^{d}\right)$. Choisissons $\alpha$ suffisamment petit pour que $A p^{d}-\alpha^{d} O\left(p^{\bar{d}}\right) \geq c p^{d}$ où $c$ est une constante strictement positive. Comme $H$ est décroissante, $D(q) \geq 0$ pour tout $q$, donc 


$$
\sum_{1 \leq p \leq N}|E(p)| \geq \sum_{1 \leq p \leq N-2} D(p)\left(A p^{d}-\alpha^{d} O\left(p^{d}\right)\right) .
$$

D'où l'inégalité

$$
\sum_{1 \leq p \leq N}|E(p)| \geq c \sum_{1 \leq p \leq N-2} D(p) p^{d} .
$$

2) Soit $I_{p}$ l'intervalle $\left[-f(p) p^{-1 / d}, f(p) p^{-1 / d}\right]$. Nous avons $E(p) \subseteq$ $\left\{T_{p}^{-1}\left(I_{p}\right)\right\}^{d}$. D'où, d'après le lemme 4 ,

$$
\begin{aligned}
|E(p) \cap E(q)| & \leq\left|\left\{T_{p}^{-1}\left(I_{p}\right)\right\}^{d} \cap\left\{T_{q}^{-1}\left(I_{q}\right)\right\}^{d}\right|=\left|\left\{T_{p}^{-1}\left(I_{p}\right) \cap T_{q}^{-1}\left(I_{q}\right)\right\}^{d}\right| \\
& \leq\left\{\left|I_{p}\right| \cdot\left|I_{q}\right|+O\left(\min \left(\left|I_{p}\right| \frac{p \wedge q}{p},\left|I_{q}\right| \frac{p \wedge q}{q}\right)\right)\right\}^{d} \\
& \leq 2^{d}\left\{\left|I_{p}\right| \cdot\left|I_{q}\right|\right\}^{d}+2^{d}\left\{O\left(\min \left(\left|I_{p}\right| \frac{p \wedge q}{p},\left|I_{q}\right| \frac{p \wedge q}{q}\right)\right)\right\}^{d} .
\end{aligned}
$$

Estimons les deux termes de cette dernière expression après sommation sur $1 \leq p, q \leq N$.

3) On a

$$
\sum_{1 \leq p, q \leq N}\left(\left|I_{p}\right| \cdot\left|I_{q}\right|\right)^{d}=\left\{\sum_{1 \leq q \leq N}\left|I_{q}\right|^{d}\right\}^{2}=\left\{\sum_{1 \leq q \leq N} 2^{d} q^{-1} f(q)^{d}\right\}^{2} .
$$

4) De plus,

$$
\begin{aligned}
& \sum_{1 \leq p, q \leq N} O\left(\min \left(\left|I_{p}\right| \frac{p \wedge q}{p},\left|I_{q}\right| \frac{p \wedge q}{q}\right)\right)^{d} \\
& =\sum_{1 \leq p \leq q \leq N} O\left(\left|I_{q}\right| \frac{p \wedge q}{q}\right)^{d} \leq \sum_{1 \leq p \leq q \leq N} O\left(f(q) q^{-1 / d} \frac{p \wedge q}{q}\right)^{d} \\
& =O\left(\sum_{1 \leq p \leq q \leq N} f(q)^{d} q^{-1-d}(p \wedge q)^{d}\right) \\
& \leq O\left(\sum_{1 \leq q \leq N} f(q)^{d} q^{-1-d} \sum_{1 \leq p \leq q}(p \wedge q)^{d}\right) \\
& \leq O\left(\sum_{1 \leq q \leq N} f(q)^{d} q^{-1-d} \sum_{m \mid q} m^{d} \sum_{k \leq q / m} 1\right) \\
& \leq O\left(\sum_{1 \leq q \leq N} f(q)^{d} q^{-1-d} \sum_{m \mid q} m^{d} q / m\right) \\
& =O\left(\sum_{1 \leq q \leq N} f(q)^{d} q^{-d} \sum_{m \mid q} m^{d-1}\right)=O\left(\sum_{1 \leq q \leq N}(f(q) / q)^{d} \sigma_{d-1}(q)\right) .
\end{aligned}
$$


Posons $R(p)=\sum_{1 \leq q \leq p} \sigma_{d-1}(q), R(-1)=0$. Une transformation d'Abel montre que

$$
\sum_{1 \leq p \leq N} f(p)^{d} p^{-d} \sigma_{d-1}(p)=\sum_{1 \leq p \leq N-2} R(p) D(p)+f\left(N^{\prime}\right)^{d} N^{\prime-d} R\left(N^{\prime}\right)
$$

où $N^{\prime}=N-1$ ou $N$. Lorsque $d \geq 2$, avec le lemme 5 nous obtenons

$$
\sum_{1 \leq q \leq N}(f(q) / q)^{d} \sigma_{d-1}(q)=O\left(\sum_{1 \leq q<N-2} D(q) q^{d}\right)+f\left(N^{\prime}\right)^{d} O(1) .
$$

5) Nous avons

$$
\begin{aligned}
\sum_{1 \leq q<N-2} D(q) q^{d} & =\sum_{1 \leq q<N-2}\left(\frac{f(q)^{d}}{q^{d}}-\frac{f(q+2)^{d}}{(q+2)^{d}}\right) q^{d} \\
& =\sum_{1 \leq q<N-2} \frac{f(q)^{d}}{q^{d}} q^{d}-\sum_{3 \leq q<N} \frac{f(q)^{d}}{q^{d}}(q-2)^{d} \\
& =\sum_{3 \leq q \leq N-2}\left(\frac{f(q)}{q}\right)^{d}\left(q^{d}-(q-2)^{d}\right) \\
& +f(1)^{d}-\frac{f\left(N^{\prime}\right)^{d}}{N^{\prime}}\left(N^{\prime}-2\right)^{d} .
\end{aligned}
$$

Comme $f(n) \rightarrow 0$ quand $n \rightarrow \infty$, pour $N$ assez grand nous avons

$$
\sum_{1 \leq q<N-2} D(q) q^{d} \geq \sum_{3 \leq q \leq N-2} \frac{f(q)^{d}}{q^{d}}\left(d(q-2)^{d-1} \cdot 2\right) \geq c \sum_{3 \leq q \leq N-2} \frac{f(q)^{d}}{q}
$$

où $c$ est une constante strictement positive. Par conséquent, d'après 1 ),

$$
\sum_{p \geq 1}|E(p)|=\infty
$$

et d'après 1) et 3),

$$
\sum_{1 \leq p, q \leq N}\left(\left|I_{p}\right| \cdot\left|I_{q}\right|\right)^{d}=O\left(\left\{\sum_{1 \leq p \leq N}|E(p)|\right\}^{2}\right) .
$$

De plus, 1) et 4) montrent que

$$
\sum_{1 \leq p, q \leq N} O\left(\min \left(\left|I_{p}\right| \frac{p \wedge q}{p},\left|I_{q}\right| \frac{p \wedge q}{q}\right)\right)^{d}=O\left(\left\{\sum_{1 \leq p \leq N}|E(p)|\right\}^{2}\right),
$$

d'où

$$
\sum_{1 \leq p, q \leq N}|E(p) \cap E(q)| \leq C\left\{\sum_{1 \leq p \leq N}|E(p)|\right\}^{2},
$$

ce qui achève la démonstration. 


\section{Démonstration du théorème 2}

1) Prolongeons la fonction $g$ à $\mathbb{R}^{+}$en une fonction décroissante; nous avons $\int^{\infty} g(t) d t<\infty$. Le changement de variable $t=\frac{1}{C} \ln s(C$ est la constante apparaissant dans le théorème 1) donne

$$
\int^{\infty} g\left(\frac{1}{C} \ln s\right) \frac{d s}{s}<\infty .
$$

En posant $\widetilde{g}(s)=g\left(\frac{1}{C} \ln s\right) / s$, nous obtenons $\int^{\infty} \widetilde{g}(s) d s<\infty$ et $\sum_{q>0} \widetilde{g}(q)$ $<\infty$. Le théorème 3 montre alors que pour presque tout $\theta \in \mathbb{T}^{1}$, et pour $n$ assez grand, nous avons

$$
\left(\frac{r_{n-1}(\theta)}{r_{n}(\theta)}\right)^{d} \leq \frac{1}{q_{n}(\theta) \widetilde{g}\left(q_{n}(\theta)\right)} .
$$

Grâce au théorème 1 nous obtenons

$$
b_{n+1}=\left(\frac{r_{n-1}(\theta)}{r_{n}(\theta)}\right)^{d} \leq \frac{1}{q_{n}(\theta) \widetilde{g}\left(q_{n}(\theta)\right)}=\frac{1}{g\left(\frac{1}{C} \ln q_{n}(\theta)\right)} \leq \frac{1}{g(n)} .
$$

2) Prolongeons $h$ à $\mathbb{R}^{+}$en une fonction décroissante; nous avons $\int^{\infty} h(t) d t$ $=\infty$. Lagarias a montré dans [5] qu'il existe une constante $c>0$ telle que

$$
\forall \theta \in \mathbb{T}^{d}, \forall n \in \mathbb{N}, \quad q_{n} \geq e^{c n} .
$$

Le changement de variable $t=\frac{1}{c} \ln s$ donne

$$
\int^{\infty} h\left(\frac{1}{c} \ln s\right) \frac{d s}{s}=\infty
$$

En posant $\widetilde{h}(s)=h\left(\frac{1}{c} \ln s\right) / s$, nous obtenons $\int^{\infty} \widetilde{h}(s) d s=\infty$ et $\sum_{q>0} \widetilde{h}(q)$ $=\infty$. Le théorème 3 montre alors que pour presque tout $\theta \in \mathbb{T}^{1}$ il existe une infinité de $n$ tels que

$$
\left(\frac{r_{n-1}(\theta)}{r_{n}(\theta)}\right)^{d} \geq \frac{1}{q_{n}(\theta) \widetilde{h}\left(q_{n}(\theta)\right)}
$$

D'après la minoration de Lagarias pour une infinité de $n$,

$$
b_{n+1}=\left(\frac{r_{n-1}(\theta)}{r_{n}(\theta)}\right)^{d} \geq \frac{1}{q_{n}(\theta) \widetilde{h}\left(q_{n}(\theta)\right)}=\frac{1}{h\left(\frac{1}{c} \ln q_{n}(\theta)\right)} \geq \frac{1}{h(n)} .
$$

6. Démonstration du théorème 4. C'est une simple adaptation de la démonstration du théorème 3 . Soit $\alpha$ vérifiant la même condition que dans la démonstration précédente. Choisissons $f$ telle que $f(n) \leq \alpha / 2, f$ décroit vers 0 et $\sum_{n \geq 0} f(n)^{d} / n=\infty$. Soit $x$ est un élément de $E(n)$. Choisissons $y \in B(n)$ tel que $d(x, y) \leq(\alpha / 2) n^{-1-1 / d}$. Nous avons

$$
\|n x\| \leq(\alpha / 2) n^{-1 / d}
$$


et, pour tout $m \in\{1, \ldots, n-1\}$,

$$
\begin{aligned}
\|m x\| & =\|m(y+x-y)\| \geq \alpha n^{-1 / d}-\|m(x-y)\| \\
& >\alpha n^{-1 / d}-\frac{\alpha}{2} n^{-1 / d}=\frac{\alpha}{2} n^{-1 / d} .
\end{aligned}
$$

L'entier $n$ est donc une meilleure approximation de $x$. Comme $\sum_{n \geq 1} f(n)^{d} / n$ $=\infty$, l'ensemble $A$ est de mesure 1 et par définition de $A$ nous avons

$$
\forall x \in A \quad \liminf _{n \rightarrow \infty} q_{n}(x) r_{n-1}(x)^{d} \geq\left(\frac{\alpha}{2}\right)^{d} .
$$

7. Démonstration du théorème 5. C'est encore une adaptation de la même méthode mais il faut remplacer le lemme 1. Ce lemme donne une minoration du nombre de sous-groupes bien répartis de $(\mathbb{Z} / n \mathbb{Z})^{k}$, alors qu'une minoration du nombre de sous-groupes mal répartis est nécessaire (voir le corollaire 3 plus loin). Un théorème de Dirichlet affirme l'existence, pour tout réel $x$ et tout entier $N$, d'un entier $q \in\{1, \ldots, N-1\}$ tel que $\|q x\|<1 / N$. La proposition 7.1 est un complément partiel de ce résultat, elle donne une minoration de la mesure de

$$
\left\{x \in \mathbb{T}^{d}: \exists k \in\{1, \ldots, N\},\|k x\| \leq r\right\}
$$

lorsque $N r^{d} \leq 1$.

Lemme 6. Soit $(\Omega, \mathcal{T}, \mu)$ un espace mesuré. Si $\left(A_{p}\right)_{1 \leq p \leq k}$ est une suite finie de parties mesurables, alors

$$
\mu\left(\bigcup_{1 \leq p \leq k} A_{p}\right) \geq \frac{\left(\sum_{1 \leq q \leq k} \mu\left(A_{q}\right)\right)^{2}}{\sum_{1 \leq p, q \leq k} \mu\left(A_{p} \cap A_{q}\right)} .
$$

Démonstration. On peut déduire cette inégalité du lemme 3 mais on peut aussi la prouver directement à partir de l'inégalité de Cauchy-Schwarz qui est l'ingrédient essentiel du lemme 3. Posons $E=\bigcup_{1 \leq i \leq k} A_{i}$; d'après l'inégalité de Cauchy-Schwarz nous avons

$$
\begin{aligned}
\left(\sum_{1 \leq i \leq k} \mu\left(A_{i}\right)\right)^{2} & =\left(\int_{E} \sum_{1 \leq i \leq k} 1_{A_{i}}(x) d \mu(x)\right)^{2} \\
& \leq\left(\int_{E} d \mu(x)\right) \int_{E}\left(\sum_{1 \leq i \leq k} 1_{A_{i}}(x)\right)^{2} d \mu(x) \\
& =\mu(E) \int_{E}\left(\sum_{1 \leq i, j \leq k} 1_{A_{i}}(x) 1_{A_{j}}(x)\right) d \mu(x) \\
& =\mu(E) \sum_{1 \leq i, j \leq k} \mu\left(A_{i} \cap A_{j}\right) .
\end{aligned}
$$


Proposition 7.1. Soient $r$ un réel appartenant à l'intervalle $[0,1 / 2]$ et $N$ un entier tels que $N r^{d} \leq 1$. Appelons E l'ensemble

$$
\left\{x \in \mathbb{T}^{d}: \exists k \in\{1, \ldots, N\},\|k x\| \leq r\right\} .
$$

(1) E est une réunion de boules de rayons supérieurs à $r / N$.

(2) $|E| \geq c N r^{d}$ où c ne dépend que de d.

Démonstration. D'après le lemme 5 nous avons $\sum_{1 \leq n \leq N} \sigma_{k}(n)=$ $O\left(N^{k+1}\right)$ pour $k \geq 1$. Si $k=0$ alors $\sigma_{0}(n)=d(n)$ et $\sum_{1 \leq n \leq N} d(n)=$ $O(N \ln N)$; ce facteur $\ln N$ oblige à distinguer les cas $d=1$ et $d \geq 2$.

Supposons $d=1$. Appelons $A_{k}=\left\{x \in \mathbb{T}^{1}: \exists m \in\{0, \ldots, k-1\}\right.$, $m \wedge k=1$ et $d(x, m / k) \leq r / k\}$ et $E_{k}=\left\{x \in \mathbb{T}^{1}: \exists m \in\{0, \ldots, k-1\}\right.$, $d(x, m / k) \leq r / k\}$. Nous avons $\bigcup_{1 \leq k \leq N} A_{k}=A=\bigcup_{1 \leq k \leq N} E_{k}=E$ et chaque $E_{k}$ est la réunion de $k$ intervalles de longueurs $2 r / k$. Il reste à minorer $|E|$; pour cela minorons $|A|$. Soit $k^{\prime}<k \leq N$. Reproduisons un argument du livre de Sprindžuk ([9]) pour estimer la mesure de $A_{k} \cap A_{k^{\prime}}$. Il est clair que

$$
\left|A_{k} \cap A_{k^{\prime}}\right| \leq 2 \frac{r}{k} N\left(k, k^{\prime}\right)
$$

où $N\left(k, k^{\prime}\right)$ est le nombre de couples $\left(a, a^{\prime}\right)$ tels que

$$
\left|\frac{a}{k}-\frac{a^{\prime}}{k^{\prime}}\right| \leq \frac{r}{k}+\frac{r}{k^{\prime}}, \quad a \wedge k=a^{\prime} \wedge k=1, \quad 0<a<k, 0<a^{\prime}<k^{\prime} .
$$

Si l'égalité $a k^{\prime}-a^{\prime} k=m$ est vraie pour un certain $m$, alors $d=k \wedge k^{\prime}$ divise $m$, et en posant $k=j d, k^{\prime}=j^{\prime} d, m=l d$, nous obtenons $a j^{\prime}-a^{\prime} j=l$. Cela montre que si $\left(b, b^{\prime}\right)$ est un autre couple tel $b k^{\prime}-b^{\prime} k=m$ alors

$$
a=b+i j, \quad a^{\prime}=b^{\prime}+i j^{\prime},
$$

où $i$ est un entier. Le nombre total de couples $\left(a, a^{\prime}\right)$ appartenant à $\{1, \ldots$, $k-1\} \times\left\{1, \ldots, k^{\prime}-1\right\}$ tels que $a k^{\prime}-a^{\prime} k=m$ est donc inférieur à $d$.

Comme $a \wedge k=a^{\prime} \wedge k=1$ et $\left|a / k-a^{\prime} / k^{\prime}\right| \leq r / k+r / k^{\prime}$ nous avons

$$
1 \leq|m| \leq r\left(k+k^{\prime}\right) \text {. }
$$

Nous ne devons considérer que les $m$ multiples de $d$, donc

$$
\begin{aligned}
N\left(k, k^{\prime}\right) & \leq \frac{2 r\left(k+k^{\prime}\right)}{d} d=2 r\left(k+k^{\prime}\right), \\
\left|A_{k} \cap A_{k^{\prime}}\right| & \leq 4 r\left(k+k^{\prime}\right) \frac{r}{k} \leq 8 r^{2} .
\end{aligned}
$$

Comme $\sum_{1 \leq p \leq n} \phi(p) \geq c_{1} n^{2}$, une transformation d'Abel montre que

$$
\sum_{1 \leq p \leq n} \frac{\phi(p)}{p} \geq c_{2} n .
$$


Finalement, l'inégalité $N \leq 1 / r$ et le lemme 6 montrent que

$$
\begin{aligned}
|A| & \geq \frac{\left\{\sum_{1 \leq k \leq N}\left|A_{k}\right|\right\}^{2}}{\sum_{1 \leq k, k^{\prime} \leq N}\left|A_{k} \cap A_{k^{\prime}}\right|} \geq \frac{\left\{\sum_{1 \leq k \leq N}(\phi(k) / k)(2 r)\right\}^{2}}{\left\{\sum_{1 \leq k, k^{\prime} \leq N} 8 r^{2}\right\}+2 N r} \\
& \geq \frac{c_{2} r^{2} N^{2}}{8 N^{2} r^{2}+2 N r} \geq \frac{c_{2} r^{2} N^{2}}{10 N r}=c N r .
\end{aligned}
$$

Supposons $d \geq 2$. Pour $1 \leq k \leq N$, désignons par $F_{k}$ l'ensemble $\{x \in$ $\left.\mathbb{T}^{d}:\|k x\| \leq r\right\}$. Nous avons $\left|\bar{F}_{k}\right|=(2 r)^{d}$. Comme $F_{k}=\left(E_{k}\right)^{d}$, le lemme 4 montre que

$$
\begin{aligned}
\left|F_{k} \cap F_{k^{\prime}}\right| & =\left|E_{k} \cap E_{k^{\prime}}\right|^{d} \leq\left(\left|E_{k}\right| \cdot\left|E_{k^{\prime}}\right|+O\left(\left|E_{k}\right| \frac{k \wedge k^{\prime}}{k}\right)\right)^{d} \\
& \leq 2^{d}\left(\left(\left|E_{k}\right| \cdot\left|E_{k^{\prime}}\right|\right)^{d}+O\left(\left|E_{k}\right| \frac{k \wedge k^{\prime}}{k}\right)^{d}\right) \\
& \leq 2^{3 d} r^{2 d}+C r^{d}\left(\frac{k \wedge k^{\prime}}{k}\right)^{d}
\end{aligned}
$$

où $C$ ne dépend que de $d$. On a

$$
\begin{aligned}
\sum_{1 \leq k^{\prime} \leq k \leq N}\left(\frac{k \wedge k^{\prime}}{k}\right)^{d} & \leq \sum_{1 \leq k \leq N} \sum_{m \mid k}\left(\frac{m}{k}\right)^{d} \sum_{1 \leq l \leq k / m} 1 \\
& =\sum_{1 \leq k \leq N} \sum_{m \mid k}\left(\frac{m}{k}\right)^{d-1}=\sum_{1 \leq k \leq N} \frac{\sigma_{d-1}(k)}{k^{d-1}} .
\end{aligned}
$$

Une transformation d'Abel et le lemme 5 montrent que

$$
\sum_{1 \leq k^{\prime} \leq k \leq N}\left(\frac{k \wedge k^{\prime}}{k}\right)^{d} \leq C N
$$

où $C$ est une nouvelle constante ne dépendant que de $d$. Grâce au lemme 6 nous obtenons

$$
|E| \geq \frac{\left(\sum_{1 \leq k \leq N}\left|F_{k}\right|\right)^{2}}{\sum_{1 \leq k^{\prime}, k \leq N}\left|F_{k} \cap F_{k^{\prime}}\right|} \geq \frac{N^{2}(2 r)^{2 d}}{2^{3 d} N^{2} r^{2 d}+C r^{d} N}=\frac{N r^{d}}{2^{d} N r^{d}+C 2^{-2 d}}
$$

et comme $N r^{d} \leq 1$ cela prouve que $|E| \geq c N r^{d}$.

Lemme 7. Soient $n$ un entier et $\left(B\left(x_{i}, r_{i}\right)\right)_{1 \leq i \leq m}$ une suite finie de boules de $\mathbb{T}^{d}$ de rayons strictement supérieurs à $1 /(2 n)$. Appelons $E=$ $\bigcup_{1 \leq i \leq m} B\left(x_{i}, r_{i}\right)$ et $\mathcal{C}=\{x \in E: n x=0\}$. On a

$$
\operatorname{card}(\mathcal{C}) \geq \frac{1}{4^{d}}|E| n^{d} .
$$

Démonstration. Comme diam $\mathbb{T}^{d}=1$ nous pouvons supposer que les rayons $r_{i}$ sont inférieurs à $1 / 2$. Pour chaque $i$ désignons par $y_{i}$ le représentant 
de $x_{i}$, appartenant à $\left(\left[0,1[)^{d}\right.\right.$. Rappelons que $\mathbb{R}^{d}$ est muni de la norme sup. Posons $F=\bigcup_{1 \leq i \leq m} B\left(y_{i}, r_{i}\right)$; nous avons $|F| \geq|E|$. De plus, $F$ est inclus dans $\left(\left[-1 / 2,3 / 2[)^{d}\right.\right.$, donc tout $x$ de $E$ a au plus $2^{d}$ représentants dans $F$ et

$$
\operatorname{card}\left(F \cap\left(\frac{1}{n} \mathbb{Z}\right)^{d}\right) \leq 2^{d} \operatorname{card}(\mathcal{C}) .
$$

Minorons $A=\operatorname{card}\left(F \cap\left(\frac{1}{n} \mathbb{Z}\right)^{d}\right)$.

Soient $B$ une boule $\mathbb{R}^{d}$ de rayon $r$ strictement supérieur à $1 /(2 n)$, et $B=I_{1} \times \ldots \times I_{d}$ où $I_{1}, \ldots, I_{d}$ sont des intervalles de longueurs $2 r$. Chaque intervalle $I_{j}$ a une longueur strictement supérieure à $1 / n$, donc chaque intervalle $I_{j}$ est inclus dans la réunion $\bigcup_{x \in I_{j} \cap(1 / n) \mathbb{Z}} B(x, 1 / n)$, par conséquent

$$
B \subset \bigcup_{x \in B \cap[(1 / n) \mathbb{Z}]^{d}} B(x, 1 / n) .
$$

D'où

$$
F \subset \bigcup_{x \in F \cap[(1 / n) \mathbb{Z}]^{d}} B(x, 1 / n)
$$

et $|E| \leq|F| \leq \operatorname{card}\left(F \cap[(1 / n) \mathbb{Z}]^{d}\right)(2 / n)^{d} \leq(4 / n)^{d} \operatorname{card}(\mathcal{C})$.

Proposition 7.2. Soient $n$ et $N$ deux entiers et $s$ un réel positif. Supposons $2 N \leq s \leq n / 2$ et $N s^{d} \leq n^{d}$. Appelons $\mathcal{E}$ l'ensemble

$$
\left\{x \in(\mathbb{Z} / n \mathbb{Z})^{d}: \exists k \in\{1, \ldots, N\}, d(0, k x) \leq s\right\} .
$$

On a $\operatorname{card}(\mathcal{E}) \geq c N s^{d}$, où c est une constante qui ne dépend que de $d$.

Démonstration. Posons $E=\left\{x \in \mathbb{T}^{d}: \exists k \in\{1, \ldots, N\}, d(0, k x) \leq\right.$ $s / n\}$. Identifions $(\mathbb{Z} / n \mathbb{Z})^{d}$ à $G=\left\{x \in \mathbb{T}^{d}: n x=0\right\}$ et $\mathcal{E}$ à $\mathbb{E}=G \cap E$. La proposition 7.1 montre que $E$ est une réunion de boules de rayons supérieurs à $s /(n N) \geq 2 / n$ et $|E| \geq c N(s / n)^{d}$, donc d'après le lemme précédent nous avons

$$
\operatorname{card}(\mathcal{E}) \geq \frac{n^{d}}{4^{d}} c N\left(\frac{s}{n}\right)^{d}=c^{\prime} N s^{d} .
$$

COROLlaire 3. Soient $n$ un entier et $\alpha$ un réel compris entre $4 n^{-1+1 / d}$ et 1 . Appelons $\mathcal{D}$ l'ensemble

$$
\begin{aligned}
\left\{x=\left(x_{1}, x^{\prime}\right) \in(\mathbb{Z} / n \mathbb{Z})^{d}:\right. & \operatorname{ordre}\left(x_{1}\right)=n \text { et } \\
& \left.\exists k \in\{1, \ldots, n-1\}, d(0, k x) \leq \alpha n^{1-1 / d}\right\} .
\end{aligned}
$$

Pour $n$ assez grand on a

$$
\operatorname{card}(\mathcal{D}) \geq c \alpha^{d} \phi(n) n^{d-1},
$$

où c est une constante strictement positive qui ne dépend que de $d$. 
Démonstration. Posons

$\mathcal{E}=\left\{x=(1, y) \in(\mathbb{Z} / n \mathbb{Z})^{d}: \exists k \in\{1, \ldots, n-1\}, d(0, k x) \leq \alpha n^{1-1 / d}\right\}$.

Remarquons que

$$
\mathcal{D}=\bigcup_{u \in \mathbb{Z} / n \mathbb{Z}, \operatorname{ordre}(u)=n} u \mathcal{E},
$$

d'où card $\mathcal{D}=\phi(n) \operatorname{card}(\mathcal{E})$. Pour minorer card $(\mathcal{E})$ utilisons la proposition précédente en dimension $d-1$ avec $s=\alpha n^{1-1 / d}$ et $N=[s / 2]$. Il est clair que

$$
\{1\} \times\left\{y \in(\mathbb{Z} / n \mathbb{Z})^{d-1}: \exists k \in\{1, \ldots, N\}, d(0, k y) \leq s\right\} \subset \mathcal{E} .
$$

Les hypothèses de la proposition 7.2 sont satisfaites car pour $n$ assez grand

$$
n / 2 \geq n^{1-1 / d} \geq s \geq 2 N \quad \text { et } \quad N s^{d-1} \leq \alpha^{d} n^{d(1-1 / d)} \leq n^{d-1},
$$

donc nous avons

$$
\operatorname{card}\left\{y \in(\mathbb{Z} / n \mathbb{Z})^{d-1}: \exists k \in\{1, \ldots, N\}, d(0, k y) \leq s\right\} \geq c N s^{d-1} .
$$

De plus $\alpha \geq 4 n^{-1+1 / d}$, donc $s \geq 4$ et $N \geq s / 4$. Finalement

$$
\operatorname{card}\left\{y \in(\mathbb{Z} / n \mathbb{Z})^{d-1}: \exists k \in\{1, \ldots, N\}, d(0, k y) \leq s\right\} \geq \frac{c}{4} s^{d}=\frac{c}{4} \alpha^{d} n^{d-1} .
$$

Fin de la démonstration du théorème 5. Soient $\alpha$ et $\beta$ deux réels tels que $\alpha \in] 0,1 / 4\left[\right.$ et $\alpha>4 \beta$. Pour $n \in \mathbb{N}^{*}$, soient

$$
\begin{aligned}
G(n) & =\left\{x \in \mathbb{T}^{d}: n x=0\right\} \\
B(n) & =\left\{a=\left(a_{1}, \ldots, a_{d}\right) \in G(n): \text { ordre }\left(a_{1}\right)=n\right. \\
\left.\qquad n^{-1 / d} \leq r(\langle a\rangle) \leq \alpha n^{-1 / d}\right\} & \\
E(n) & =\left\{x \in \mathbb{T}^{d}: d(x, B(n))<(\beta / 2) n^{-1-1 / d}\right\} \\
E^{\prime}(n) & =\left\{x \in \mathbb{T}^{d}: d(x, B(n)) \leq \beta n^{-1-1 / d}\right\} .
\end{aligned}
$$

Soit $x$ appartenant à $E(n)$. Un argument de la démonstration du théorème 3 montre que $n$ est une meilleure approximation de $x$. De plus par définition de $E(n)$ il existe $q<n$ tel que

$$
\|q x\| \leq \alpha n^{-1 / d}+q \frac{\beta}{2} n^{-1-1 / d} \leq 2 \alpha n^{-1 / d} .
$$

Donc, si $m$ est la meilleure approximation de $x$ précédant $n$, nous avons

$$
n\|m x\|^{d} \leq n\|q x\|^{d} \leq(2 \alpha)^{d} .
$$

Ainsi un élément $x$ de $\mathbb{T}^{d}$ appartenant à une infinité de $E(n)$ vérifie

$$
\liminf _{n \rightarrow \infty} q_{n}(x) r_{n-1}^{d}(x) \leq(2 \alpha)^{d},
$$

pour démontrer le théorème il suffit donc de prouver que pour tout $\alpha>0$ la mesure de $\limsup _{n \rightarrow \infty} E(n)$ est 1 . Cela se démontre comme le théorème 3 car, d'après le lemme 1 et le corollaire 3 , nous avons

$$
\operatorname{card}(B(n)) \geq \phi(n)\left(c \alpha^{d} n^{d-1}-2^{2 d-1} \beta^{d} n^{d-2} \sigma_{1}(n)\right) .
$$


8. Preuve de la proposition 1.1. Pour un entier strictement positif $k$ posons :

$$
\begin{aligned}
& x(k)= \begin{cases}\left(2^{-a(k)}, 0,0\right) & \text { si } k \equiv 1 \text { ou } 2 \bmod 6 \\
\left(0,3^{-a(k)}, 0\right) & \text { si } k \equiv 3 \text { ou } 4 \bmod 6, \\
\left(0,0,5^{-a(k)}\right) & \text { si } k \equiv 5 \text { ou } 0 \bmod 6\end{cases} \\
& y(k)=\sum_{i=1}^{k} x(i),
\end{aligned}
$$

où $a$ est une fonction strictement croissante de $\mathbb{N}$ dans $\mathbb{N}$. Posons

$$
\theta=\sum_{i=0}^{\infty} x(i) .
$$

La série $\sum_{i=0}^{\infty} x(i)$ converge car la fonction $a$ est strictement croissante. Dans la suite des conditions supplémentaires seront imposées à la fonction $a$; ces conditions sont réalisées pour des fonctions croissant suffisamment rapidement. Pour tout $k \geq 1, y(k)$ est un élément de $\mathbb{T}^{3}$ d'ordre fini, appelons $n(k)$ son ordre. Nous avons pour $k \geq 1$,

$$
n(k)= \begin{cases}2^{a(k-4)} 3^{a(k-2)} 5^{a(k)} & \text { si } k \equiv 0 \bmod 6 \\ 2^{a(k)} 3^{a(k-3)} 5^{a(k-1)} & \text { si } k \equiv 1 \bmod 6 \\ 2^{a(k)} 3^{a(k-4)} 5^{a(k-2)} & \text { si } k \equiv 2 \bmod 6 \\ 2^{a(k-1)} 3^{a(k)} 5^{a(k-3)} & \text { si } k \equiv 3 \bmod 6 \\ 2^{a(k-2)} 3^{a(k)} 5^{a(k-4)} & \text { si } k \equiv 4 \bmod 6 \\ 2^{a(k-3)} 3^{a(k-1)} 5^{a(k)} & \text { si } k \equiv 5 \bmod 6\end{cases}
$$

Construisons la fonction $a(k)$ par récurrence. Supposons $a(k-1)$ construit. Envisageons le cas $k \equiv 1 \bmod 6$, les autres cas se traitent de la même façon. Une petite perturbation de $y(k-1)$ ne change pas les meilleures approximations de $y(k-1)$. Plus précisement, il existe un nombre $\varepsilon_{k}>0$ tel que, pour tout $x$ de $\mathbb{T}^{3}$ de norme $\leq \varepsilon_{k}$, les deux propriétés suivantes aient lieu :

$$
\begin{gathered}
\forall q \in\{1, \ldots, n(k-1)\}, \quad q \text { meilleure approximation de } y(k-1) \Leftrightarrow \\
\quad q \text { meilleure approximation de } y(k-1)+x, \\
\forall m<n(k-1), \quad\|m(y(k-1)+x)\| \leq 2\|m y(k-1)\| .
\end{gathered}
$$

Choisissons $a(k)$ tel que $\|x(k)\|=2^{-a(k)} \leq \frac{1}{2} \varepsilon_{k}$. Nous pouvons supposer que la suite $\left(\varepsilon_{k}\right)$ vérifie $\varepsilon_{k+1} \leq \frac{1}{2} \varepsilon_{k}$. Comme $\theta=\lim _{k \rightarrow \infty} y(k)$ nous obtenons $\|\theta-y(k-1)\| \leq \varepsilon_{k}$ pour tout $k \geq 2$. La condition (1) montre que la suite $(n(k))$ est une sous-suite de la suite des meilleures approximations de $\theta$ et que les meilleures approximations de $\theta$ inférieures ou égales à $n(k-1)$ sont celles 
de $y(k-1)$. La condition (2) montre que si $q$ est une meilleure approximation de $\theta$ strictement inférieure à $n(k-1)$ alors $\|q \theta\| \leq 2\|q y(k-1)\|$.

Evaluons $q_{m} r_{m-1} r_{m-2} r_{m-3}$ :

Lemme 8. Soit $x=\left(x_{1}, x_{2}, x_{3}\right) \in \mathbb{T}^{3}$. Si le sous-groupe $\left\langle\left(x_{2}, x_{3}\right)\right\rangle$ a un cardinal inférieur à $N$, alors pour tout $n$ tel que $q_{n}(x)$ soit définie on a $q_{n}(x) r_{n-1}(x) \leq N$.

Soit $q_{m}$ la plus petite meilleure approximation de $\theta$ appartenant à l'intervalle $] n(6 p), n(6 p+2)]$.

Posons $k=6 p$. Le lemme montre que

$$
\begin{aligned}
q_{m}(y(k+1)) r_{m-1}(y(k+1)) & \leq 3^{a(k-2)} 5^{a(k)} \\
q_{m-1}(y(k)) r_{m-2}(y(k)) & \leq 2^{a(k-4)} 3^{a(k-2)} \\
q_{m-2}(y(k)) r_{m-3}(y(k)) & \leq 2^{a(k-4)} 3^{a(k-2)}
\end{aligned}
$$

comme $q_{m-1}=n(k)<n(k+1)$ et $q_{m-2}<n(k)$, la condition (2) montre que

$$
\begin{gathered}
q_{m}(\theta) r_{m-1}(\theta) \leq 2 \cdot 3^{a(k-2)} 5^{a(k)}, \\
q_{m-1}(\theta) r_{m-2}(\theta) \leq 2 \cdot 2^{a(k-4)} 3^{a(k-2)}, \\
q_{m-2}(\theta) r_{m-3}(\theta) \leq 2 \cdot 2^{a(k-4)} 3^{a(k-2)} .
\end{gathered}
$$

Nous avons $q_{m-1}=n(k)$ et $q_{m-2} \geq n(k-1)$, donc

$$
\begin{aligned}
& q_{m}(\theta) r_{m-1}(\theta) r_{m-2}(\theta) r_{m-3}(\theta) \\
& \leq\left(2 \cdot 3^{a(k-2)} 5^{a(k)}\right)\left(n(k)^{-1} 2 \cdot 2^{a(k-4)} 3^{a(k-2)}\right)\left(n(k-1)^{-1} 2 \cdot 2^{a(k-4)} 3^{a(k-2)}\right) \\
& \leq 8 \cdot 3^{a(k-2)} 5^{a(k)} 2^{-a(k-4)} 3^{-a(k-2)} 5^{-a(k)} 2^{a(k-4)} 3^{a(k-2)} \\
& \quad \times 2^{-a(k-4)} 3^{-a(k-2)} 5^{-a(k-1)} 2^{a(k-4)} 3^{a(k-2)} \\
& =8 \cdot 3^{a(k-2)} 5^{-a(k-1)} .
\end{aligned}
$$

Soit $q_{p}(\theta)$ une meilleure approximation de $\theta$ comprise entre $q_{m}$ et $n(k+2)$. Le lemme montre que

$$
q_{p}(y(k+2)) r_{p-1}(y(k+2)) \leq 3^{a(k-2)} 5^{a(k)},
$$

de plus $r_{p-2}(\theta) \leq r_{m-2}(\theta)$ et $r_{p-3}(\theta) \leq r_{m-3}(\theta)$, le calcul précédent montre donc que

$$
q_{p}(\theta) r_{p-1}(\theta) r_{p-2}(\theta) r_{p-3}(\theta) \leq 8 \cdot 3^{a(k-2)} 5^{-a(k-1)} .
$$

En choisissant $a(k-1)$ suffisamment grand devant $a(k-2)$, le membre de gauche est aussi petit que l'on veut. Un raisonnement identique montre le même résultat pour les meilleures approximations appartenant aux intervalles $] n(6 p+2), n(6 p+4)]$ et $] n(6 p+4), n(6 p+6)]$. 


\section{Références}

[1] N. Chevallier, Distances dans la suite des multiples d'un point du tore à deux dimensions, Acta Arith. 74 (1996), 47-59.

[2] -, Meilleures approximations d'un élément du tore $\mathbb{T}^{2}$ et géométrie de la suite des multiples de cet élément, ibid. 78 (1996), 19-35.

[3] G. H. Hardy and E. M. Wright, An Introduction to the Theory of Numbers, Oxford Univ. Press, 1938.

[4] J. C. Lagarias, Some new results in simultaneous Diophantine approximation, in: Proc. of the Queen's Number Theory Conference 1979, P. Ribenboim (ed.), Queen's Papers in Pure and Appl. Math. 54, Queen's Univ., 1980, 453-474.

[5] -, Best simultaneous Diophantine approximations, I. Growth rates of best approximations denominators, Trans. Amer. Math. Soc. 72 (1982), 545-554.

[6] -, Best simultaneous Diophantine approximations, II. Behavior of consecutive best approximations, Pacific J. Math. 102 (1982), 61-88.

[7] -, Geodesic multidimensional continued fractions, Proc. London Math. Soc. (3) 69 (1994), 464-488.

[8] W. M. Schmidt, Diophantine Approximation, Lecture Notes in Math. 785, Springer, 1980.

[9] V. G. Sprindžuk, Metric Theory of Diophantine Approximations, Winston, Washington, D.C., 1979.

Université de Haute Alsace

4, rue des Frères Lumière

68093 Mulhouse, France

E-mail: n.chevallier@univ-mulhouse.fr

Reçu le 2.4.1999

et révisé le 29.5.2000 